\title{
Preoperative diagnosis of multiple intraductal tubulopapillary neoplasms of the pancreas: dynamic imaging features from endoscopic ultrasound
}

A 60-year-old man with recurrent pancreatitis was admitted to our hospital for further examination and treatment. Abdominal computed tomography highlighted multiple cystic lesions in the pancreas ( Fig.1a). Magnetic resonance imaging revealed atrophic pancreatic parenchyma and segmentally dilated pancreatic duct ( $\triangleright$ Fig.1 b). The diagnosis of intraductal papillary mucinous neoplasm (IPMN) or intraductal tubulopapillary neoplasm (ITPN) was proposed. Subsequent endoscopic ultrasound (EUS) showed multiple cystic lesions with solid tumors inside extending along the dilated main pancreatic duct ( $\triangleright$ Fig. 1 c). The intraductal tumor was surrounded by pancreatic fluid in the head of the pancreas, representing the "cork-of-winebottle sign;" however, the hypoechoic tumors connecting with anechoic fluids were displayed as two different colors in the dilated duct, indicating the "2-tone duct sign" ( $\triangleright$ Video 1$)$. Based on the

a
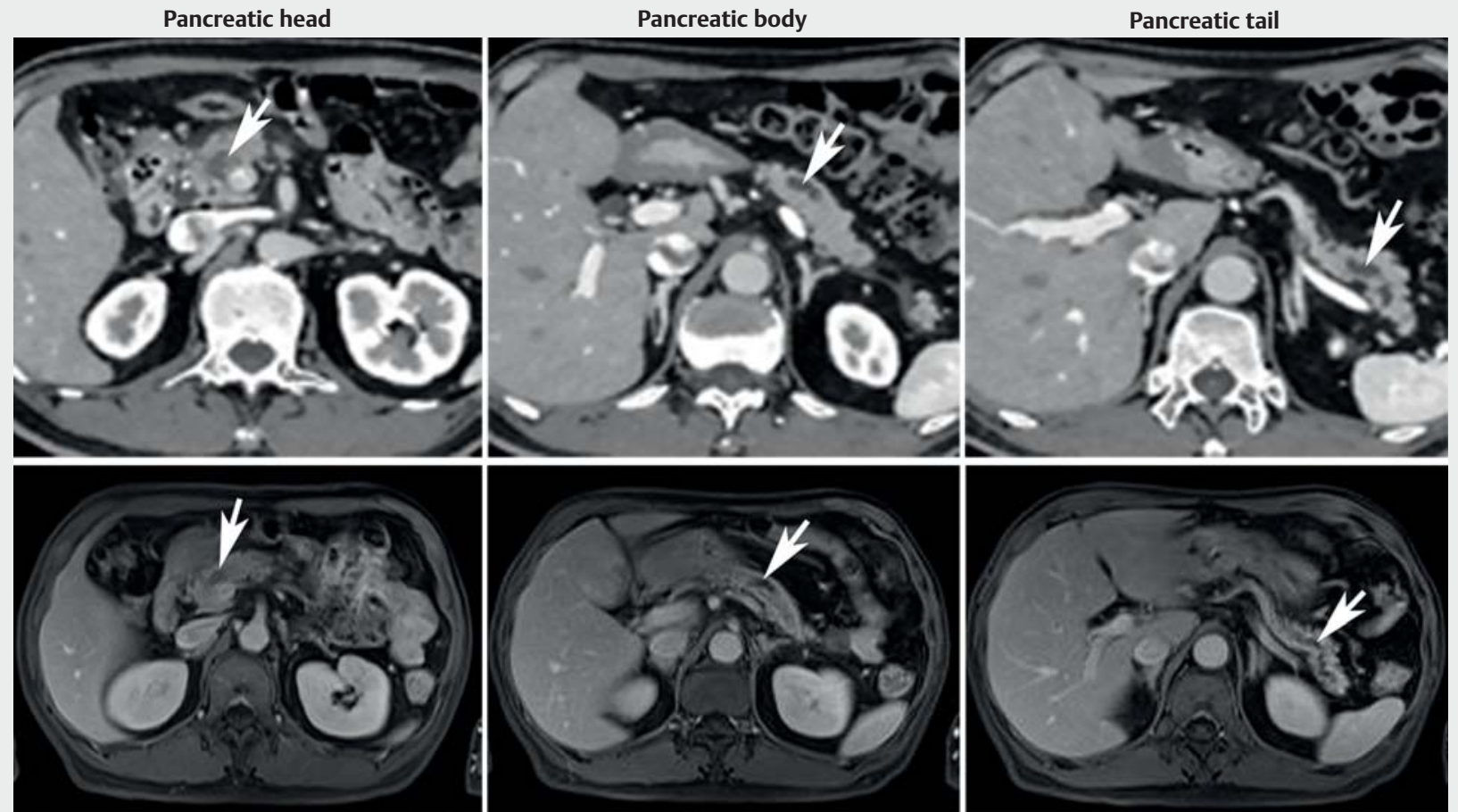

b
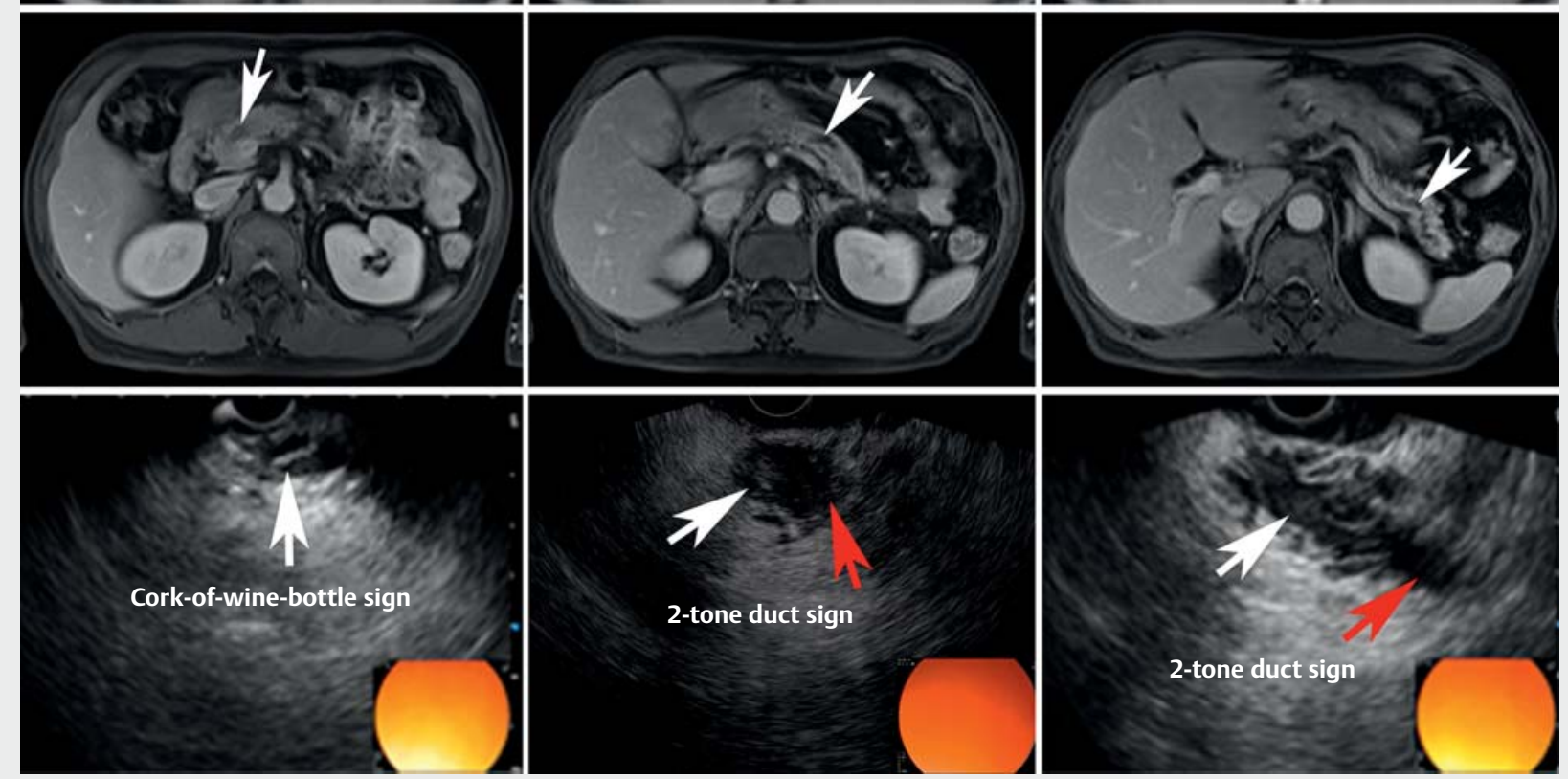

- Fig. 1 Imaging manifestations of intraductal tubulopapillary neoplasm in a 60-year-old man. a Transverse dynamic computed tomography showed cystic lesions (arrows) in the dilated pancreatic duct. b Transverse magnetic resonance imaging also indicated multiple cystic lesions along the dilated duct in the head, body, and tail of the pancreas (arrows). c Transverse endoscopic ultrasound showed several intraductal tumors slightly protruding from the segmentally dilated pancreatic ducts, in which the "2-tone duct sign" (white and red arrows) and "cork-ofwine-bottle sign" (arrow) can be seen. 

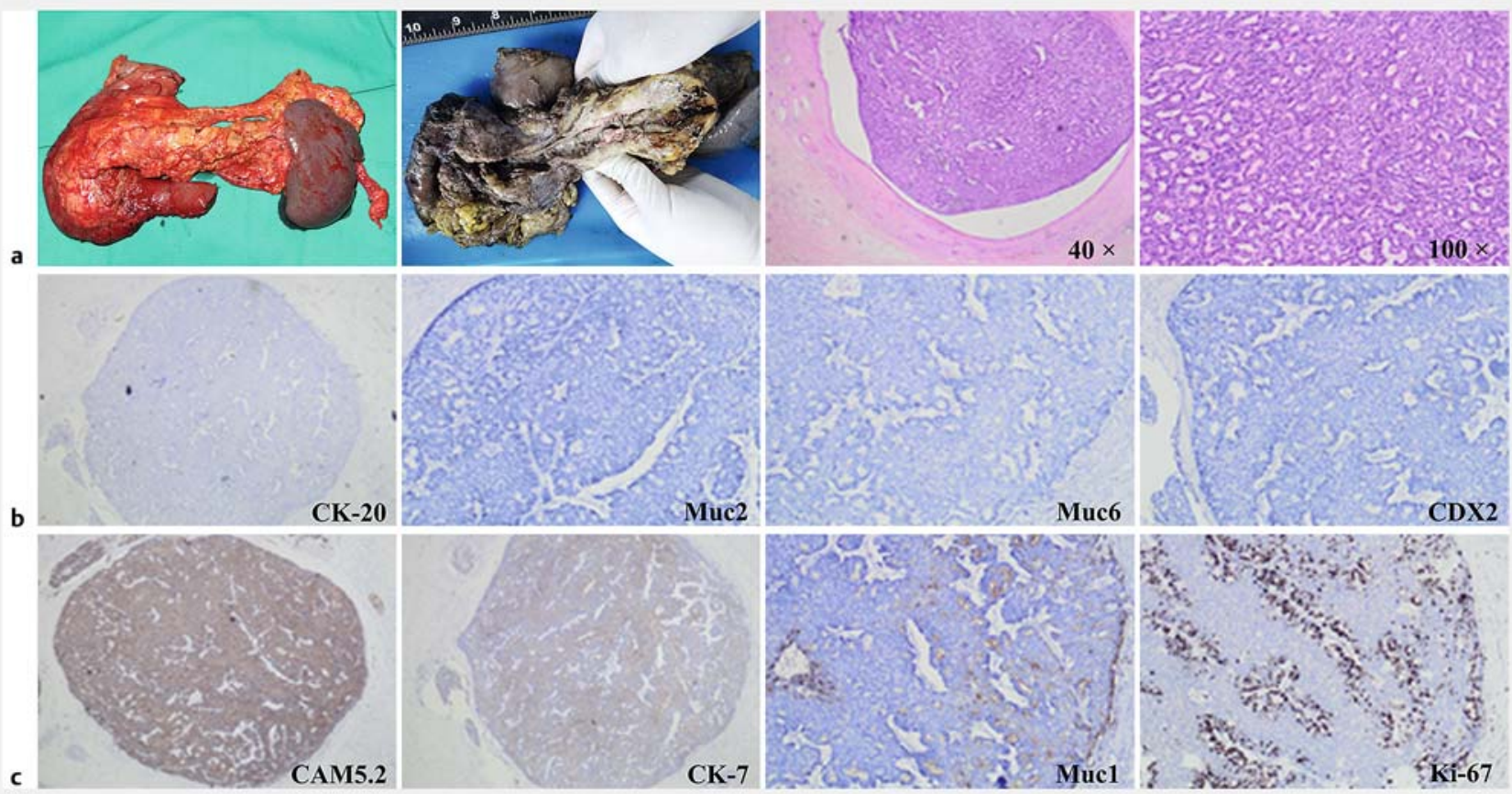

- Fig. 2 Histopathologic characteristics of intraductal tubulopapillary neoplasm in a 60-year-old man. a Gross specimen presenting solid tumors without mucin located in the dilated pancreatic duct, and the histopathologic slides stained with hematoxylin and eosin showing the dilated main pancreatic duct containing a solid tumor with tubulopapillary structures. $\mathbf{b}$ Immunohistochemical staining revealed that the tumor was negative for CK-20, MUC2, MUC6, and CDX2. c Immunohistochemistry demonstrated positive staining for CAM5.2, CK-7, and MUC1. The proliferative index of Ki-67 reached $70 \%$.

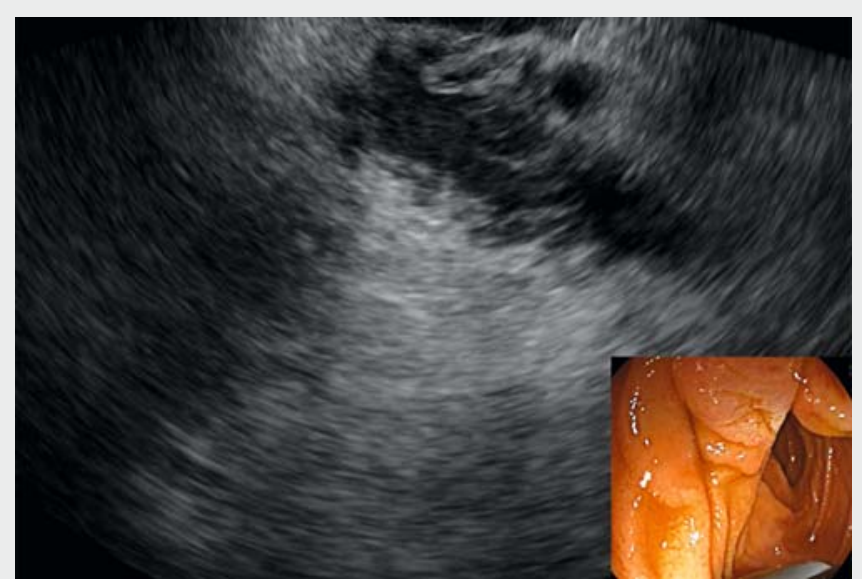

Video 1 Preoperative diagnosis of multiple intraductal tubulopapillary neoplasms of the pancreas based on the dynamic imaging features from endoscopic ultrasound, consisting of the "2-tone duct sign" and the "cork-of-wine-bottle sign."

above two typical imaging findings [1,2], ITPN was reliably diagnosed preoperatively using EUS; thus, surgery was recommended.

The resected specimen was identified with solid tumors in the dilated pancreat- ic duct manifesting as soft and grayish yellow. No mucin was observed. Histopathology showed the tumor consisted of tubulopapillary structures with cylindrical cells and high grade dysplasia ( $>$ Fig. 2a). On immunohistochemistry, the tu- mor was negative for MUC2, MUC6, CK20, and $\operatorname{CDX} 2$ ( $/$ Fig. 2 b), and positive for CK7, CAM5.2, and MUC-1 (\$ Fig. 2c). The Ki-67 index reached $70 \%$ ( $>$ Fig. 2 c). The findings were consistent with an ITPN diagnosis.

As a rare pancreatic neoplasm, ITPN is very difficult to diagnose before surgery even with a combination of imaging modes [3]. We report the dynamic imaging features of multiple ITPNs of the pancreas on EUS, including the "2-tone duct sign" and "cork-of-wine-bottle sign." The limitation of this study is that EUS-guided biopsy may provide more information in the differential diagnosis between IPMN and ITPN [4, 5].

Endoscopy_UCTN_Code_CCL_1AZ_2AM

\section{Competing interests}

The authors declare that they have no conflict of interest. 
Wei-hui Liu ${ }^{1,{ }^{*},}$ Xiao-ju Su ${ }^{2,{ }^{*}, \text { Xu Fang }}{ }^{3}$, Hui Jiang $^{4}$, Shi-wei Guo ${ }^{5}$, Kai-xuan Wang ${ }^{2}$

1 Department of Gastroenterology and Hepatology, Sichuan Academy of Medical Sciences and Sichuan Provincial People's Hospital, Chengdu, China

2 Department of Gastroenterology, Changhai Hospital, Second Military Medical University and Naval Medical University, Shanghai, China

3 Department of Radiology, Changhai Hospital, Second Military Medical University and Naval Medical University, Shanghai, China

4 Department of Pathology, Changhai Hospital, Second Military Medical University and Naval Medical University, Shanghai, China

5 Department of General Surgery, Changhai Hospital, Second Military Medical University and Naval Medical University, Shanghai, China

\section{Corresponding author}

\section{Kai-xuan Wang, MD, PhD}

Department of Gastroenterology, Changhai Hospital, Second Military Medical University and Naval Medical University, No.168 Changhai Road, Yangpu District, Shanghai 200433, China

Fax: +86-28-86571251

wangkaixuan224007@163.com
[1] Motosugi U, Yamaguchi H, Furukawa T et al. Imaging studies of intraductal tubulopapillary neoplasms of the pancreas: 2-tone duct sign and cork-of-wine-bottle sign as indicators of intraductal tumor growth. J Comput Assist Tomogr 2012; 36: 710-717

[2] Zhang J, Ren S, Wang J et al. Imaging findings of intraductal tubulopapillary neoplasm (ITPN) of the pancreas: two case reports and literature review. Medicine 2019; 98: e14426

[3] Kuan LL, Dennison AR, Garcea G. Intraductal tubulopapillary neoplasm of the pancreas and bile duct: a review. Pancreas 2020; 49: 498-502

[4] Huang DC, Bilal M, Gonzalez RS et al. Not every cyst is an intraductal papillary mucosal neoplasm: a case of intraductal tubulopapillary neoplasm. Gastrointest Endosc 2020; 92: 967-968

[5] Kovacevic B, Latorre Ano P, Toxvaerd A et al. Intraductal tubulopapillary neoplasm of the pancreas diagnosed by endoscopic ultrasonography-guided fine needle aspiration. Endoscopy 2017; 49: E266-E267
Bibliography

Endoscopy 2021; 53: E345-E347

DOI 10.1055/a-1290-7182

ISSN $0013-726 \mathrm{X}$

published online 11.11 .2020

(c) 2020. Thieme. All rights reserved.

Georg Thieme Verlag KG, Rüdigerstraße 14 ,

70469 Stuttgart, Germany

\section{ENDOSCOPY E-VIDEOS}

https://eref.thieme.de/e-videos

回回 Endoscopy E-Videos is a free access online section, reporting 靣: on interesting cases and new techniques in gastroenterological endoscopy. All papers include a high quality video and all contributions are freely accessible online.

This section has its own submission website at https://mc.manuscriptcentral.com/e-videos

\footnotetext{
* These authors contributed equally to this work.
} 